\title{
A case of hepatitis C-associated osteosclerosis: accelerated bone turnover controlled by pulse steroid therapy
}

\author{
Nobuhiro Miyamura', Shuhei Nishida', Mina Itasaka', Hirofumi Matsuda', \\ Takeshi Ohtou ${ }^{2}$, Yasuhiro Yamaguchi ${ }^{3}$, Daisuke Inaba ${ }^{4}$, Sadahiro Tamiya ${ }^{5}$ and \\ Tetsuo Nakano 4 \\ 1Departments of Diabetes and Endocrinology, ${ }^{2}$ Gastroenterology, ${ }^{3}$ Neurology, and ${ }^{4}$ Orthopedic Surgery, \\ Tamana Central Hospital, Tamana, Japan, and ${ }^{5}$ Department of General and Community Medicine, \\ Kumamoto University Hospital, Kumamoto, Japan
}

\author{
Correspondence \\ should be addressed \\ to N Miyamura \\ Email \\ miyamura@tamana-chp.jp
}

\section{Summary}

Hepatitis $\mathrm{C}$-associated osteosclerosis (HCAO), a very rare disorder in which an extremely rapid bone turnover occurs and results in osteosclerosis, was acknowledged in 1990s as a new clinical entity with the unique bone disorder and definite link to chronic type $C$ hepatitis, although the pathogenesis still remains unknown. Affected patients suffer from excruciating deep bone pains. We report the 19th case of HCAO with diagnosis confirmed by bone biopsy, and treated initially with a bisphosphonate, next with corticosteroids and finally with direct acting antivirals (DAA: sofosbuvir and ribavirin) for HCV infection. Risedronate, $17.5 \mathrm{mg} /$ day for 38 days, did not improve the patient's symptoms or extremely elevated levels of bone markers, which indicated hyper-bone-formation and coexisting hyper-bone-resorption in the patient. Next, intravenous methylprednisolone pulse therapy followed by high-dose oral administration of prednisolone evidently improved them. DAA therapy initiated after steroid therapy successfully achieved sustained virological response, but no additional therapeutic effect on them was observed. Our results strongly suggested that the underlying immunological alteration is the crucial key to clarify the pathogenesis of HCAO. Bone mineral density of lumbar vertebrae of the patient was increased by $14 \%$ in four-month period of observation. Clarification of the mechanisms that develop osteosclerosis in HCAO might lead to a new therapeutic perspective for osteoporosis.

\section{Learning points:}

- HCAO is an extremely rare bone disorder, which occurs exclusively in patients affected with HCV, of which only 18 cases have been reported since 1992 and pathogenesis still remains unclear.

- Pathophysiology of HCAO is highly accelerated rates of both bone formation and bone resorption, with higher rate of formation than that of resorption, which occur in general skeletal leading to the diffuse osteosclerosis with severe bone pains.

- Steroid therapy including intravenous pulse administration in our patient evidently ameliorated his bone pains and reduced elevated values of bone markers. This was the first successful treatment for HCAO among cases reported so far and seemed to propose a key to solve the question for its pathogenesis.

- The speed of increase in the bone mineral content of the patient was very high, suggesting that clarification of the mechanism(s) might lead to the development of a novel therapy for osteoporosis. 


\section{Background}

Hepatitis C-associated osteosclerosis (HCAO) is a very rare disorder in which an extremely rapid bone turnover occurs, resulting in generalized osteosclerosis. Affected patients suffer from excruciating deep bone pain in appendicular skeleton. This condition occurs exclusively in patients affected with $\mathrm{HCV}$, of which only 18 cases have been reported since 1992 despite the high prevalence of hepatitis $\mathrm{C}$ worldwide, and its etiopathogenesis still remains unclear (1). Here we report a new case, with diagnosis confirmed by bone biopsy and treated initially with a bisphosphonate, next with steroid therapy including intravenous pulse administration, and finally with direct acting antivirals (DAA) aiming at sustained virological response (SVR). We also describe detailed data including patient's symptoms, biochemical values and bone markers through the clinical course.

\section{Case presentation}

A 50-year-old Japanese man was admitted to our hospital because of long-standing pains mainly in his lower extremities. His body mass index was $19.5 \mathrm{~kg} / \mathrm{m}^{2}$ and blood pressure was $153 / 88$. He had been well until approximately 2.5 years before this admission, when he experienced a sudden onset of pain in both legs. Initially, thermalgia occurred along his tibias, and it gradually extended to thigh bones, hip joints and lumbar area in 2 years. Skeletal radiographs showed a diffuse osteosclerosis of thickening diaphyseal cortices, pelvic bones and spinal vertebrae. A radiologic appearance in which the endplates are densely sclerotic (sandwich vertebral body), which is distinctive for osteopetrosis, was absent. Paget's disease of bone was ruled out because no focal bone deformation or sign of bone fracture was observed. An abdominal ultrasound examination revealed several gallstones sized up to $8 \mathrm{~mm}$ within the gallbladder.

There was no family history of bone disease. History of exposure to fluoride or specific heavy metals was also absent. He was first told that he had been infected with $\mathrm{HCV}$ in his age 20, however, he had been asymptomatic and never been treated until this episode. Although he had no history of drug abuse, blood transfusion, acupuncture or tattoo, he had once pierced his ear using a non-sterilized needle.

\section{Investigation}

Laboratory data revealed low-normal serum calcium level with remarkable hypocalciuria, high levels of both
intact-PTH and 1,25-dihydroxy vitamin D with low-normal level of 25-hydroxy vitamin D. Very high levels of both bone formation markers and bone resorption markers were present (data at the initial evaluations are summarized in Table 1, 1st column, hospital day -20 to +30 ).

Bone scintigraphy using 99m-Tc-HMDP showed an enhanced radionuclide uptake without any focal abnormality (Fig. 1). Neither parathyroid gland swelling nor abnormal 99m-Tc-MIBI uptake was detected by ultrasound imaging or parathyroid scintigraphy. Dualenergy X-ray absorptiometry (DEX) revealed remarkably elevated levels of generalized bone mineral density (BMD) (Table 2, 1st column).

Bone biopsy specimen taken from iliac crest after double tetracycline labeling was analyzed (Fig. 2). Parametric values of bone volume, trabecular thickness, osteoid volume, osteoid thickness, osteoid surface and eroded surface were enormously elevated (Table 1, 1st column). A number of osteoclasts were also present. Immature bone mineralization occurred in many trabecula suggesting exceedingly rapid bone formation (Fig. 3). These observations suggested that the bone turnover rate was highly increased, in which, bone formation exceeded resorption leading to a positive balance and were consistent with the results of biochemical markers of bone metabolism. Other disorders causing osteosclerosis were inconsistent with the case. HCAO, a quite rare disorder for which there are no established diagnostic criteria, seemed the most appropriate diagnosis. To evaluate the severity of pain, universal pain assessment tool was used with arbitrary pain scale unit of 0 (no pain) to 10 (worst possible pain), and the patient recorded the score every one hour or two during daytime of the hospitalization period. The pain score at the initial evaluation was located at around 7-8.

\section{Treatment}

After obtaining informed consent of the patient and approval of Institutional Ethics Committee, treatment was initiated with risedronate, $17.5 \mathrm{mg} /$ day. Supplementation with calcium L-aspartate hydrate $(2400 \mathrm{mg} /$ day $)$ and alphacalcidol $(1.0 \mu \mathrm{g} /$ day $)$ was required to maintain normocalcemia soon after the regimen was started. We stopped risedronate on regimen day 38 because a preferable effect was not observed in bone pain or biochemical markers of bone (Fig. 4A and B), and hypocalciuria was remarkable despite supplementation. Calcium carbonate $(3000 \mathrm{mg} / \mathrm{day})$ was added to the supplementation aiming at increasing urinary calcium 
Table 1 Clinical data summary of serum and urine, bone histomorphometry and HCV viral parameters.

\begin{tabular}{l}
\hline \\
Treatment \\
Serum \\
Calcium (mg/dL) \\
Phosphate (mg/dL) \\
Albumin (mg/dL) \\
Creatinine (mg/dL) \\
AST (IU/L) \\
ALT (IU/L) \\
Alkaline phosphatase (IU/L) \\
TSH (IU/mL) \\
GH (ng/mL) \\
IGF-1 (ng/mL) \\
PTH (pg/mL) \\
Osteocalcin (ng/mL) \\
BAP (mg/mL) \\
Total-PINP \\
TRACP-5b (U/dL) \\
25-Hydroxyvitamine D (ng/mL) \\
1,25-Dihydroxyvitamine D (pg/mL) \\
Urinary \\
NTX (nmolBCE/mmol Cr) \\
DPD (nmol/mmol Cr) \\
Calcium/creatinine ratio $(\%)$ \\
Bone histomorphometry (iliac crest) \\
Bone volume BV/TV (\%) \\
Trabecular thickness ( $\mu \mathrm{m})$ \\
Osteoid volume OV/BV (\%) \\
Osteoid surface OS/BS (\%) \\
Osteoid thickness ( $\mu \mathrm{m})$ \\
Eroded surface ES/BS (\%) \\
Mineral apposition rate ( $\mu \mathrm{m} / \mathrm{day})$ \\
Mineralizing surface MS/BS (\%) \\
Bone formation rate (mm3/mm²/year) \\
HCV antibody \\
Serotype group \\
RNA quantification (log IU/mL) \\
\hline
\end{tabular}

Reference
Range

$8.3-10.4$
$2.5-4.5$
$4.1-5.1$
$0.6-1.2$
$13-34$
$7-37$
$106-350$
$0.35-4.94$
$<2.47$
$87-245$
$10-65$
$2.5-13$
$3.7-20.9$
$18-74$
$170-590$
$7-41$
$20-60$
$13-66$
$2.1-5.4$
$5-15$
$25.7 \pm 6.9$
$138 \pm 6.2$
$4.5 \pm 0.8$
$27 \pm 2.8$
$11.1 \pm 0.7$
$3.7 \pm 0.5$
$1.02 \pm 0.5$
$7.4 \pm 3.8$
$0.016 \pm 0.008$

\begin{tabular}{|c|c|c|c|}
\hline \multicolumn{4}{|c|}{ Hospital day } \\
\hline$-20 \sim 30$ & 130 & 190 & 280 \\
\hline $\begin{array}{l}\text { None (before } \\
\text { treatment) }\end{array}$ & $\begin{array}{l}\text { After mPSL } \\
\text { infusion }\end{array}$ & $\begin{array}{c}\text { PSL (per os) } \\
\text { Continued }\end{array}$ & $\begin{array}{c}\text { After } \\
\text { DAA therapy }\end{array}$ \\
\hline
\end{tabular}

$\begin{array}{cc}8.2 & 8.6^{*} \\ 3 & 3.5^{*} \\ 4.1 & 4 \\ 0.72 & 0.88 \\ 42 & 68 \\ 37 & 171 \\ 1529 & 1571 \\ 0.74 & - \\ 0.37 & - \\ 82 & - \\ 219 & 167^{*} \\ 40 & 21 \\ 184 & 197 \\ 1200< & 1080 \\ 1220 & 1180 \\ 11 & 13^{*} \\ 95 & 119 * \\ & \\ 900 & 286 \\ 34.2 & 14.8 \\ 0.29 & 3.6^{*}\end{array}$

$44.1(+2.7$ S.D.)

256 (+19 S.D.)

8.36 (+4.8 S.D.)

75.9 (+17 S.D.)

13.6 (+3.6 S.D.)

6.2 (+5.0 S.D.)

0.37 (-1.3 S.D.)

38.4 (+8.2 S.D.)

0.052 (+4.5 S.D.)

Reactive

Type 2

$-$

*Data were sampled under supplementation with alfacalcidol and calcium. - not measured.

BAP, bone-specific alkaline phosphatase; BS, bone surface; BV, bone volume; DAA, direct acting antivirals; DPD, deoxypiridinoline; ES, eroded surface; mPSL, methylprednisolone; MS, mineralizing surface; NTX, N-terminal telopeptide; OS, osteoid surface; OV, osteoid volume; PINP, N-terminal propeptides of procollagen type I; TRACP, tartrate-resistant acid phosphatase; TV, total volume.

excretion by phosphorus absorption, which did not seem effective (Fig. 4A and B).

Steroid administration was initiated on 100th hospital day. First, a regimen consisting of intravenous infusion of methylprednisolone (mPSL), $500 \mathrm{mg} /$ day for three days, and subsequent withdrawal for four days was repeated four times (pulse steroid therapy). Subsequent to the regimen, oral administration of prednisolone (PSL) was started, and the dose was soon increased up to $50 \mathrm{mg}$. The dose was gradually decreased down to $10 \mathrm{mg} /$ day. DAA therapy (sofosbuvir, $400 \mathrm{mg} /$ day and ribavirin, $800 \mathrm{mg} /$ day) was initiated on 195th day and continued for 84 days. Supplementation was gradually withdrawn by 240th day because the hypocalcemic tendency of the patient seemed to disappear.

\section{Outcome and follow-up}

Steroid administration revealed its impact on the symptoms and biochemical data of the patient. Pulse steroid therapy led to a notable improvement of pains of his lower extremities and decrease of ALP and a bone 


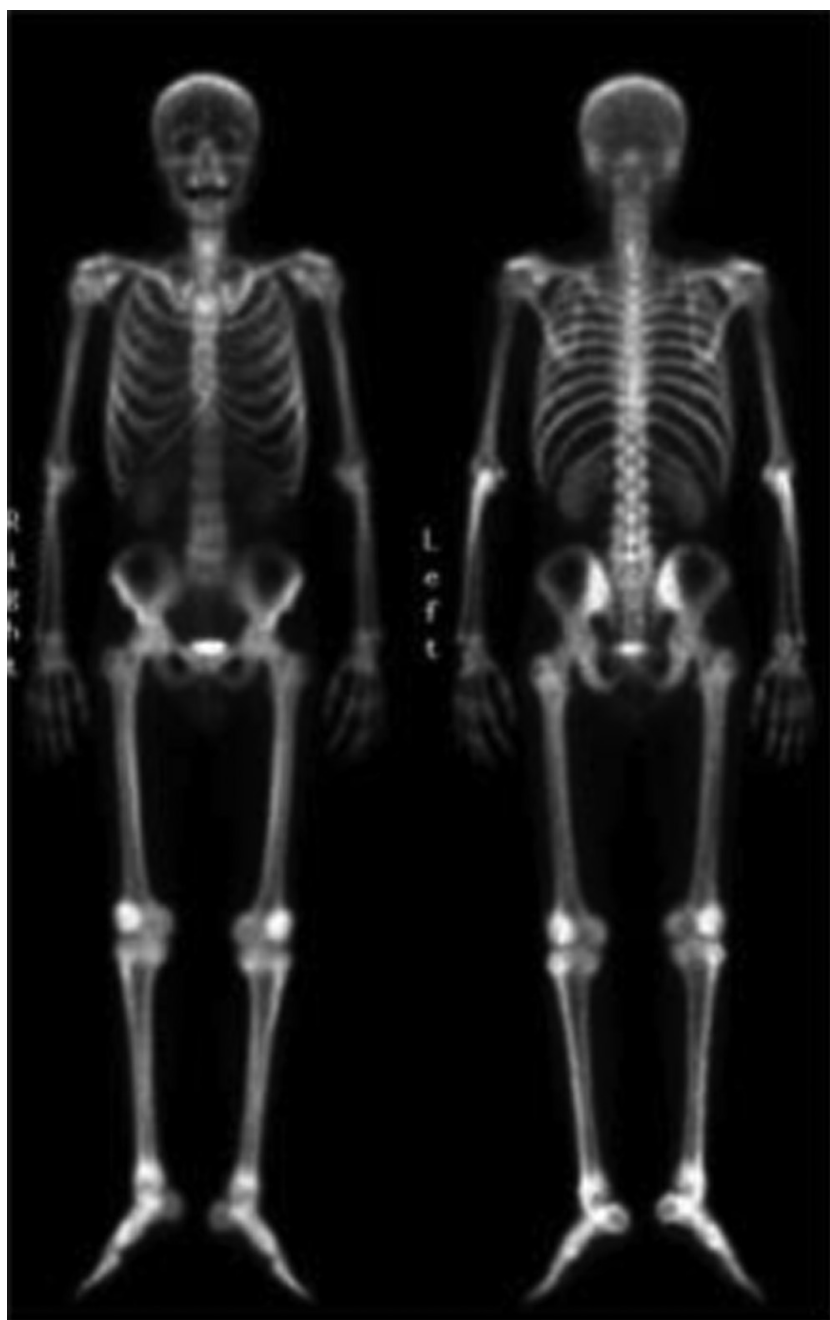

\section{Figure 1}

Bone scintigraphy using 99m-Tc-HMDP. Left panel, anterior view. Right panel, posterior view.

formation marker of BAP during infusion periods (Fig. 4A). The pain-relieving effect was weakened during each withdrawal period. Oral administration of PSL was started, and the dose was increased up to $50 \mathrm{mg}$ because his pain worsened again. Values of ALP and BAP, once decreased by mPSL therapy, became higher levels than those before. They, however, reached peak levels on around 150th

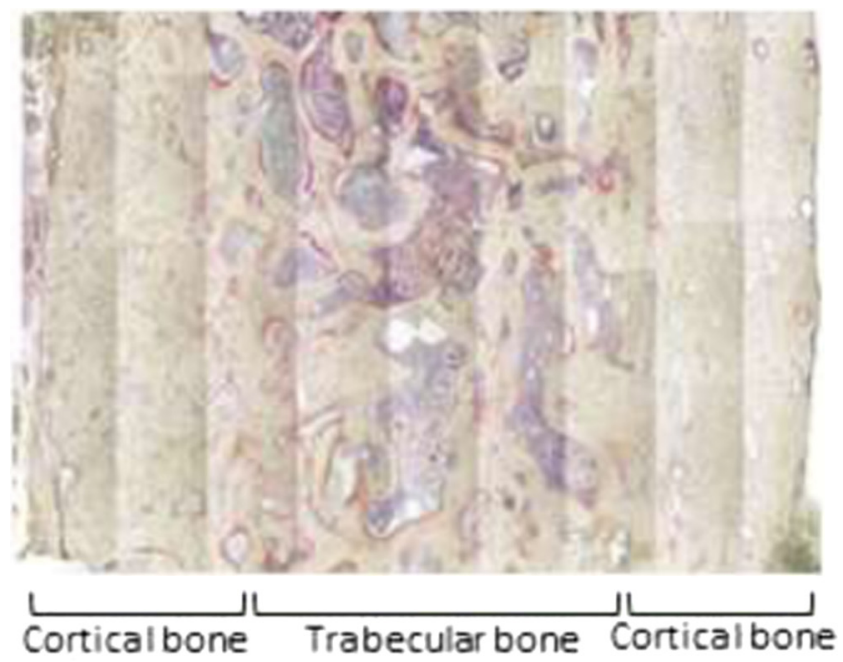

Figure 2

Bone biopsy specimen taken from iliac crest. Cortical bone zones are evidently thickened and cancellous bone zone is thinned.

hospital day, and then began to decrease linearly down to the levels around the upper limit of normal range in about 50 days (Fig. 4A). Meanwhile, pain score, which had been 6- 8 before MPSL infusion, was decreased by the treatment to the level of 3-5, but was sustained at the same level thereafter (Fig. 4A). DAA therapy successfully achieved SVR (Table 1, hospital day 280) but showed no additional effect on his symptoms or bone markers (Fig. 4A and B).

The representative markers of bone resorption and formation, urine deoxypyridinoline and serum osteocalcin respectively, were also observed through the course (Fig. 4B and Table 1). Risedronate decreased the elevated values of both markers during the initial 2 or 3 weeks but thereafter the effect seemed to disappear, so did the phosphorus absorption therapy with calcium carbonate. Pulse steroid therapy and subsequent oral administration successfully decreased the levels of both markers, which reached around the upper limit of normal range in about 10 weeks. Of note, steroid therapy seemed to aggravate hepatitis, leading to elevated levels of transaminases, which were promptly normalized with the use of DAA (Fig. 4B and Table 1, 4th column, hospital day 280).

Table 2 Initial values and changes in bone mineral density and bone mineral content.

\begin{tabular}{l} 
Measured date \\
\hline BMD total $\left(\mathrm{g} / \mathrm{cm}^{2}\right)$ (T-score) \\
Left femoral neck hip \\
Lumbar spine (L2-L4) \\
Total BMC (g) \\
Increase rate of total BMC (g/day)
\end{tabular}

\begin{tabular}{c}
\hline day 0 \\
\hline $1.446(3.8)$ \\
$1.417(4.4)$ \\
$1.513(3.9)$ \\
3184 \\
- \\
\hline
\end{tabular}

\begin{tabular}{c}
\hline day $\mathbf{1 5 5}$ \\
\hline $1.509(4.6)$ \\
$1.436(4.5)$ \\
$1.730(5.7)$ \\
3442 \\
1.66 \\
\hline
\end{tabular}

\begin{tabular}{c}
\hline day $\mathbf{2 1 6}$ \\
\hline $1.531(4.8)$ \\
$1.496(5.0)$ \\
$1.759(6.0)$ \\
3529 \\
1.43 \\
\hline
\end{tabular}

\begin{tabular}{c}
\hline day 249 \\
\hline $1.557(5.2)$ \\
$1.492(5.0)$ \\
$1.788(6.2)$ \\
3607 \\
2.36 \\
\hline
\end{tabular}

\begin{tabular}{cccc}
\cline { 1 - 1 } \cline { 1 - 1 } day $\mathbf{3 0 2}$ & & day $\mathbf{4 2 2}$ \\
\cline { 1 - 1 } $1.569(5.3)$ & & $1.594(5.6)$ \\
$1.522(5.2)$ & & $1.544(5.4)$ \\
$1.709(5.6)$ & & $1.687(5.4)$ \\
3653 & & 3689 \\
0.87 & & 0.3 \\
\hline
\end{tabular}

$\mathrm{BMC}$, bone mineral content; BMD, bone mineral density; DXA, dual-energy X-ray absorptiometry. 


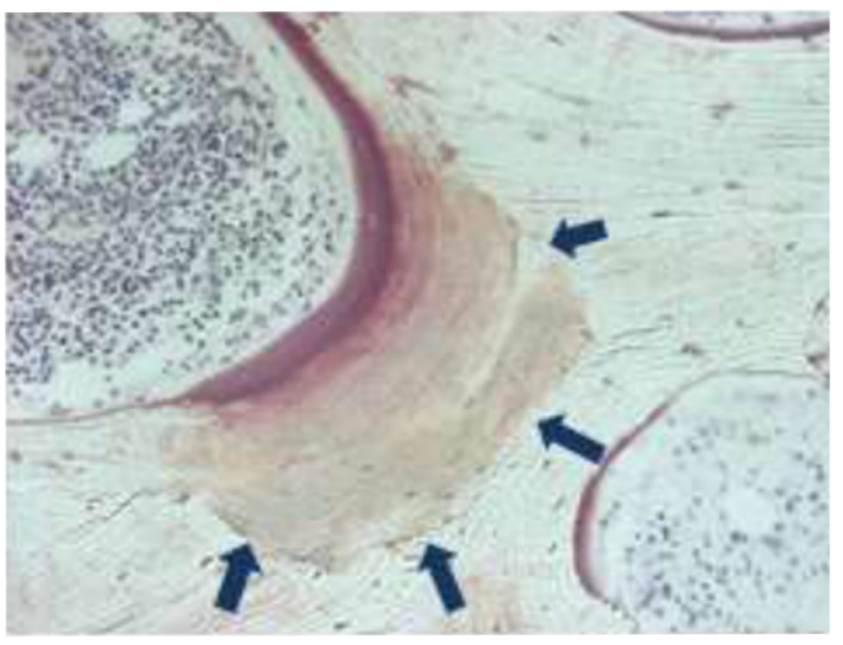

Figure 3

Bone histomorphometry of high magnification field $(\times 200)$. Arrows indicate immaturely mineralized area, which suggests highly increased bone formation rate.

DXA evaluations revealed sustained increase of BMD and bone mineral content (BMC) despite decreasing levels of bone markers. Total BMC was increased by the rate of $1.43-2.36 \mathrm{~g} /$ day since the initial evaluation until day 249 (Table 2). These values were compatible with those estimated by calcium balance, suggesting that most of the orally ingested calcium was incorporated into bone without being excreted in urine. The rate, however, slowed down thereafter to $0.87 \mathrm{~g} /$ day, and the most recent data showed further slowdown to $0.30 \mathrm{~g} /$ day in the subsequent 120 days.

The patient was gaining $870 \mathrm{mg}$ of bone mineral content daily (absolute value estimated by whole body dual-energy X-ray absorptiometry). Bone mineral content seems to consist of hydroxyapatite $\left(\mathrm{Ca}_{10}\left(\mathrm{PO}_{4}\right)_{6}(\mathrm{OH})_{2}\right)$, of which the weight percentage of calcium is $\sim 40 \%$. The value was consistent with calcium balance (oral ingestion and urinary excretion).

\section{Discussion}

The first several cases of HCAO were reported in 1992 (2). This is a very uncommon condition and can be defined as an acquired painful skeletal disorder characterized by a remarkable increase of bone mass. Osteosclerosis is characterized by nonspecific, often diffuse bony pain and tenderness over involved bones due to periosteal stretching, in the absence of joint swelling. Radiographic examination shows thickening of the long bone cortices, mainly diaphyseal cortical bone. Deterioration of bone strength has not been reported except for an elderly

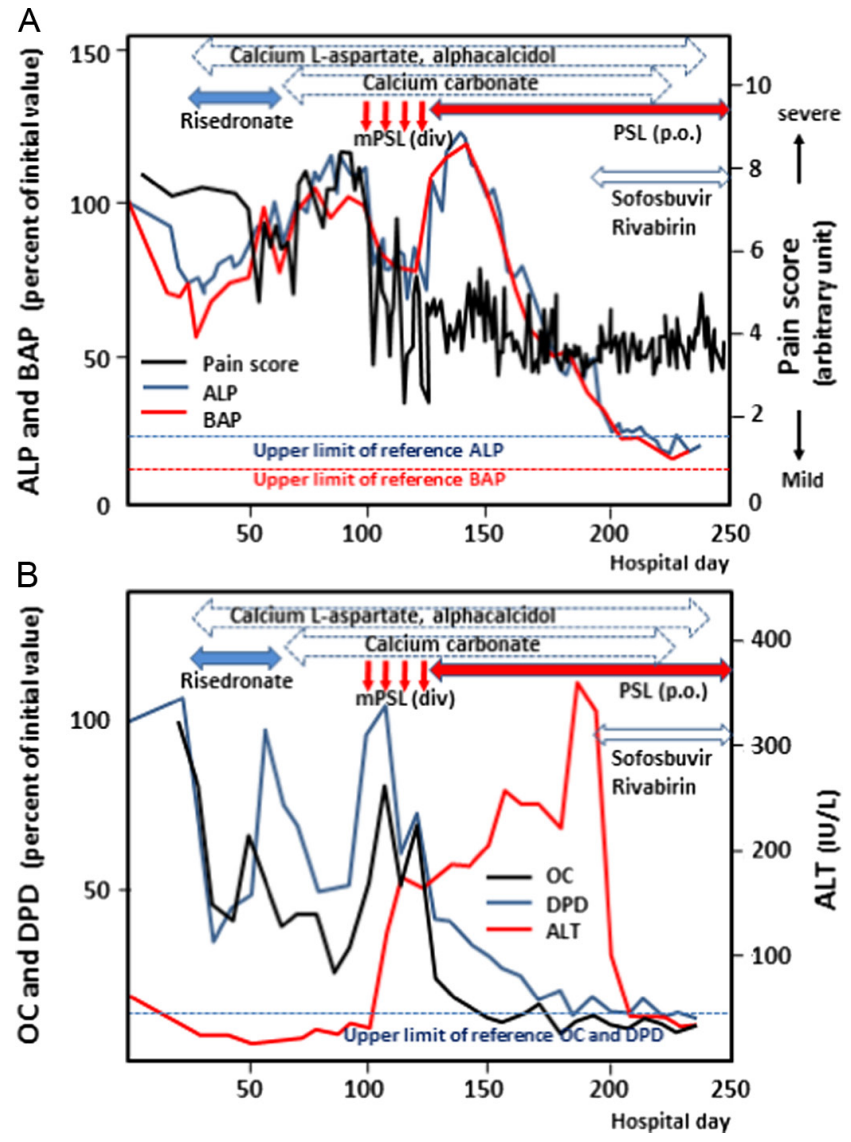

Figure 4

Clinical course of the patient during the hospitalization. Two-way arrows show the periods of treatments, red vertical arrows indicate pulse steroid therapy. (A) Pain score (arbitrary unit: 0 none -10 most severe), ALP and BAP ( $\%$ of initial value) are shown as line graphs of black, blue and red respectively. (B) OC, DPD (\% of initial value) and ALT (IU/L) are shown as line graphs of black, blue and red respectively. BAP, bone-specific alkaline phosphatase; $\mathrm{BMC}$, bone mineral content; $\mathrm{BMD}$, bone mineral density; DPD, deoxypyridinoline; DXA, dual-energy X-ray absorptiometry; $\mathrm{mPSL}$, methylprednisolone; PSL, prednisolone; OC, osteocalcin.

woman who suffered from multiple fractures after several falls 10 years after the diagnosis of HCAO (1). The pathogenesis of this rare condition still remains unclear. It has been suggested that HCV alone or in combination with other unknown factor(s) may infect and alter bone cells or their precursors in predisposed individuals. Abnormalities have been demonstrated by previous studies in the insulin-like growth factor (IGF) system (3), the receptor activator of nuclear factor kappa B ligand (RANKL) and osteoprotegerin (OPG) system imbalance $(4,5)$, and the transforming growth factor (TGF)- $\beta$-Smad signaling (6). With regards to therapeutic approaches to the disease, the hallmarks are the changes in deep, torturing bony pain, bone markers, thickening of the long bone cortices and BMD. Attempts to treat with anti-resorptive agents in at 
least five patients were ineffective, whereas symptomatic treatments may provide some benefit (1). In some cases, a partial or complete spontaneous remission of symptoms and/or osteosclerosis was observed during follow-up. Cholecystectomy in a case having xanthogranulomatous cholecystitis with multiple gallstones might have led to the declining of her elevated BMD, although characteristic bone pain was absent in the case (7). The antiviral therapy in a case using ribavirin and interferon was followed by a recovery of skeletal sclerosis (8).

The 19th case of HCAO was herein described with the detailed clinical course of the patient for almost one year. We focused on the therapeutic effect of several treatments on his bone pain, biochemical data and bone markers (Fig. 4). Bony pain was measured every one hour by a pain scale during daytime, and serum and urine were sampled almost every week. A bisphosphonate (risedronate; used in a high-dose regimen) showed only a transient partial remission. This unusual regimen of an anti-resorptive agent is recommended only for the patients with Paget's disease of bone in Japan. Our result suggested that the mechanisms that lead to accelerated bone turnover in HCAO (diffuse sclerosis) and Paget's disease (focal) differ with each other. DAA has never been challenged for HCAO treatment before this case, and ineffectiveness of DAA therapy using sofosbuvir was observed on the abnormal bone turnover and bony pain despite an achieved SVR (Fig. 4B). An argument would be that it was used after a remission achieved by steroid treatment and some positive effect of SVR might be veiled. In addition, skeletal recovery by the antiviral treatment reported previously was recognized when 7 years passed after the treatment (8).

We found an impact of intravenous pulse therapy followed by high-dose oral administration of glucocorticoids on the patient's pain and laboratory data. Among 20 cases of HCAO in the literature, steroid treatment was described only in a case, with $5 \mathrm{mg}$ /day of PSL for 2.5 months, without subjective improvement (9). Our results strongly suggested that some underlying immunological alteration is the crucial key to clarify the pathogenesis. DEX revealed increased lumbar BMD $\left(1.513 \mathrm{~g} / \mathrm{cm}^{2}\right.$ in $\mathrm{L} 2-\mathrm{L} 4$ average), which further reached $1.73 \mathrm{~g} / \mathrm{cm}^{2}$ in the subsequent 130 days since initial evaluation (Table 2). Elevated levels of both PTH and 1,25-OH vitamin D and strongly suppressed urinary calcium excretion despite low-normal 25-OH vitamin $\mathrm{D}$ and a large amount of calcium supplementation suggested that they worked in accordance to increase BMD (Table 1) and that some unknown factor(s) might regulate all of these actions. It might be that promoted bone resorption is the reaction to protect skeletal from osteosclerosis, and treatment by anti-resorptive agent could be with adverse effect. One of the most remarkable therapeutic effects for osteoporosis, reported in a clinical study using intermittent subcutaneous injections of PTH in postmenopausal women with severe osteoporosis, was an increase by $8 \%$ per year in lumbar BMD, the rate far smaller than that observed in this case (14\% per 130 days). Clarification of the mechanisms that develop osteosclerosis in HCAO might lead to a new therapeutic perspective for osteoporosis.

In conclusion, $\mathrm{HCAO}$ is a very rare disorder, and no definitive treatment has hitherto been found. We reported the first case of HCAO in which a therapy using high dose of steroid was evidently effective, proposing not only a key to solve the question for its pathogenesis but also an entrance to a new treatment strategy for bone loss syndromes in future.

\section{Declaration of interest}

The authors declare that there is no conflict of interest that could be perceived as prejudicing the impartiality of the research reported.

\section{Funding}

This research did not receive any specific grant from any funding agency in the public, commercial or not-for-profit sector.

\section{Patient consent}

Written informed consent was obtained from the patient.

\section{Author contribution statement}

All the authors have read the manuscript and have approved this article. $\mathrm{N}$ Miyamura is the author and the corresponding author of this article. S Nishida, M Itasaka, H Matsuda, T Ohtou, Y Yamaguchi, D Inaba, S Tamiya and T Nakano are clinicians who contributed to the management of this case.

\section{References}

1 Serraino C, Melchio R, Silvestri A, Borretta V, Pomero F \& Fenoglio L 2015 Hepatitis C-associated osteosclerosis: a new case with long-term follow-up and a review of the literature. Internal Medicine 54 777-783. (doi:10.2169/internalmedicine.54.3448)

2 Villareal DT, Murphy WA, Teitelbaum SL, Arens MQ \& Whyte MP 1992 Painful diffuse osteosclerosis after intravenous drug abuse. American Journal of Medicine 93 371-381. (doi:10.1016/00029343(92)90165-8)

3 Khosla S, Hassoun AAK, Baker BK, Liu F, Zein NN, Whyte MP, Reasner CA, Nippoldt TB, Tiegs RD, Hintz RL, et al. 1998 Insulinlike growth factor system abnormalities in hepatitis $\mathrm{C}$-associated 
osteosclerosis. Potential insights into increasing bone mass in adults. Journal of Clinical Investigation 101 2165-2173. (doi:10.1172/JCI1111)

4 Manganelli P, Giuliani N, Fietta P, Mancini C, Lazzaretti M, Pollini A, Quaini F \& Pedrazzoni M 2005 OPG/RANKL system imbalance in a case of hepatitis C-associated osteosclerosis: the pathogenetic key? Clinical Rheumatology 24 296-300. (doi:10.1007/ s10067-004-1031-3)

5 Enjuanes A, Ruiz-Gaspà S, Peris P, Ozalla D, Álvarez L, Combalia A, Martínez De Osaba MJ, Monega A, Pares A \& Guañabens N 2010 The effect of the alendronate on OPG/RANKL system in differentiated primary human osteoblasts. Endocrine 37 322-328. (doi:10.1007/ s12020-009-9306-8)

6 Kaji H, Naito J, Sowa H, Sugimoto T \& Chihara K 2006 Serum soluble factors induce the proliferation, alkaline phosphatase activity and transforming growth factor- $\beta$ signal in osteoblastic cells in the patient with hepatitis C-associated osteosclerosis. Experimental Clinical Endocrinology and Diabetes 114 599-604. (doi:10.1055/s-2006-924399)

7 Hataya Y, Komatsu Y, Chusho H, Kirishima T, Shintani H \& Morimoto T 2011 A case of hepatitis C-associated osteosclerosis with xanthogranulomatous cholecystitis. Internal Medicine 50 1703-1708. (doi:10.2169/internalmedicine.50.4675)

8 Javier RM, Vernejoul MC, Afif N, Kunts JL \& Sibilia J 2011 Skeletal recovery from hepatitis $\mathrm{C}$-associated osteosclerosis (HCAO) following antiviral treatment. Joint Bone Spine 78 409-411. (doi:10.1016/j. jbspin.2011.02.016)

9 Schwartz KM \& Skinner JA 2008 Hepatitis C-associated osteosclerosis: a case report. Skeletal Radiology 37 679-681. (doi:10.1007/s00256-0080471-2)

Received in final form 3 October 2016

Accepted 26 October 2016 